Research Paper

\title{
Dehydroepiandrosterone supplementation combined with Weight-Loading Whole-Body Vibration Training (WWBV) affects exercise performance and muscle glycogen storage in middle-aged C57BL/6 mice
}

\author{
Yi-Ming Chen ${ }^{1}$, Hao-Chieh Lee ${ }^{1}$, Mu-Tsung Chen ${ }^{2}$, Chi-Chang Huang ${ }^{3, 凶}$ and Wen-Chyuan Chen ${ }^{4,5, \varangle}$ \\ 1. Health Technology Collage, Jilin Sport University, Changchun 130022, Jilin, China. \\ 2. School of Liberal Education, Shih Chien University, Taipei 116, Taiwan \\ 3. Graduate Institute of Sports Science, National Taiwan Sport University, Taoyuan 33301, Taiwan \\ 4. Center for General Education, Chang Gung University of Science and Technology, Taoyuan 33301, Taiwan \\ 5. Department of Otorhinolaryngology-Head and Neck Surgery, Sleep Center, Linkou-Chang Gung Memorial Hospital, Taoyuan 33301, Taiwan
}

$\triangle$ Corresponding author: Graduate Institute of Sports Science, National Taiwan Sport University, No. 250, Wenhua 1st Rd., Guishan Township, Taoyuan County 33301, Taiwan (C.-C.H.); Center for General Education, Chang Gung University of Science and Technology; No. 250, Wenhua 1st Rd., Guishan District, Taoyuan City 33301, Taiwan (W.-C.C.). Tel.: +886-3-328-3201 (ext. 2619) (C.-C.H.); +886-3-211-8999 (ext. 5301) (W.-C.C.). Electronic addresses: john5523@ntsu.edu.tw (C.-C.H.); wcchen@gw.cgust.edu.tw (W.-C.C.).

(C) Ivyspring International Publisher. This is an open access article distributed under the terms of the Creative Commons Attribution (CC BY-NC) license (https://creativecommons.org/licenses/by-nc/4.0/). See http://ivyspring.com/terms for full terms and conditions.

Received: 2017.10.16; Accepted: 2018.02.07; Published: 2018.03.09

\begin{abstract}
Background: Adequate nutritional intake and an optimal training program are important elements of any strategy to preserve or increase muscle mass and strength during aging.

Purpose: In the current study, we investigate the effects of Dehydroepiandrosterone (DHEA), one of the most abundant circulating steroids in humans and a precursor hormone, supplementation combined with a weight-loading whole-body vibration (WWBV) on exercise performance, physical fatigue-related biochemical responses and testosterone content in middle-aged 9 months old C57BL/6 mice.

Methods: Male middle-aged C57BL/6 mice were divided into 3 groups ( $n=8$ per group) and treated for 4 weeks with the following: 1) Sedentary control (SC) with vehicle 2) DHEA supplementation (DHEA, 10.2 $\mathrm{mg} / \mathrm{kg}$ ) and 3) DHEA supplementation with WWBV training (DHEA: $10.2 \mathrm{mg} / \mathrm{kg}$; WBV: $5.6 \mathrm{~Hz}, 2 \mathrm{~mm}, 0.13 \mathrm{~g}$ ). Exercise performance was evaluated by forelimb grip strength and time to exhaustion, as well as changes in body composition and anti-fatigue levels after a 15-min swimming exercise. Fatigue-related biochemical responses of serum lactate, ammonia, glucose, creatine kinase (CK), and blood urea nitrogen (BUN) were measured following the swimming exercise. In addition, the biochemical parameters and the testosterone levels were measured at the end of the experiment.

Results: DHEA supplementation combined with WWBV training for 4 weeks significantly decreased the amount of white adipose tissue and increased the food and water intake. Additionally, WWBV+DHEA supplementation improved exercise performance, testosterone levels and glycogen contents of both liver and muscle. WWBV+DHEA supplementation also decreased serum lactate, ammonia and BUN levels, while increasing glucose levels following the 15-min swim test.
\end{abstract}

Conclusion: Taken together, our results suggest that combining the WWBV training program with DHEA supplementation could provide an anti-fatigue pharmacological effect for elderly populations.

Key words: dehydroepiandrosterone (DHEA); resistance training; weight-loading whole-body vibration (WWBV); exercise performance; glycogen.

\section{Introduction}

Muscle power has been reported to decrease with increasing age and muscle wasting is an important problem for older people [1]. Muscle strength plays an important role in determining risk for falls in older adults [2] with muscle mass declining between $3-8 \%$ each decade after 30 years of age [3]. 
Resistance training, also known as strength or weight training, has become one of the efficient forms of exercise for enhancing muscle strength, as well as for conditioning in older people. Given the role of resistance training in maintaining muscle strength and preventing muscle wasting, it is understandable that leading researchers have advocated a public health mandate for sensible resistance training [4].

DHEA supplementation is one direct way to increase levels of sex steroid hormone leading to increase in muscle mass and prevention of muscle wasting [5] in older subjects. DHEA is reversibly converted to dehydroepiandrosterone sulfate (DHEAS) [6], a precursor of sex steroid hormones. Serum DHEA levels generally decrease with aging [7] and previous study has shown that DHEA supplementation leads to improved glucose metabolism-related signaling pathway and enzyme activities in skeletal muscle [8] and insulin resistance in obese rat [9].

Whole-body vibration (WBV) is a kind of supplementary training or light-resistance training based on automatic body adaptations to rapid and repeated oscillations of a vibrating platform [10]. Studies have demonstrated several benefits of WBV, including improving muscle strength [11], increasing bone mineral density $[12,13,14]$, decreasing abdominal fat [15] and increasing hormone content [16]. Although there is considerable evidence that WBV is similar to resistance training, WBV still lacks sufficient strength stimulation. In order to resolve WBV's problem of a lack of training intensity, we combined resistance and WBV training through the use of weight-loading WBV (WWBV). In addition, we included DHEA supplementation, which has been shown to have several benefits in age-advanced subjects [14]. There has been no prior study on the effects of a combination of DHEA supplementation and WWBV training on body composition, serum biochemical indexes, exercise performance and hormone content. In this study, we combined DHEA supplementation and WWBV training for middle-age mice to investigate the beneficial synergistic effects on hormone content, muscle mass, body composition, exercise performance, biochemical profiles and pathological responses after 4 -weeks of treatment.

\section{Materials and methods}

\section{Materials, Animals, and Experiment Design}

DHEA used for supplementation in this study was obtained from General Nutrition Centers, Inc. (GNC, Pittsburgh, PA, USA). Male middle-age C57BL/6 mice (9 months old) under specific pathogen-free conditions were purchased from the
National Laboratory Animal Center (NLAC) at the National Applied Research Laboratories (Taipei, Taiwan). The mice were acclimatized to the environment and diet for one week before experimentation. All mice were provided a standard laboratory diet (No. 5001; PMI Nutrition International, Brentwood, MO, USA) and distilled water ad libitum. They were housed with a 12-h light/12-h dark cycle at room temperature $\left(24 \pm 1^{\circ} \mathrm{C}\right)$ and $50-60 \%$ humidity. The Institutional Animal Care and Use Committee (IACUC) of National Taiwan Sport University inspected all animal experiments in this study and the study conformed to the guidelines of protocol IACUC-10511 approved by the IACUC ethics committee.

The middle-aged mice were randomly assigned to 3 groups (8 mice/group): 1) Sedentary control with vehicle (SC) 2) DHEA supplementation (DHEA) and 3) Weight-loading whole-body vibration (WWBV) with DHEA supplementation (WWBV+DHEA). Food intake and water consumption were recorded daily and all animals were weighed weekly.

\section{DHEA Supplementation}

The DHEA and WWBV+DHEA groups of mice were supplemented with DHEA (oral gavage) once a day for 4 weeks at $10.2 \mathrm{mg} / \mathrm{kg} /$ day. The SC group received the same volume of distilled water equivalent to body weight. The DHEA supplementation in the WWBV+DHEA group was given 30 minutes after WWBV training. The recommended use of DHEA for humans is about 50 mg per single intake with a normal diet and exercise program. The mouse DHEA dose $(10.2 \mathrm{mg} / \mathrm{kg})$ used in this study was converted from a human equivalent dose on the basis of body surface area by the following formula from the US Food and Drug Administration [17]: Assuming a human weight of 60 $\mathrm{kg}$, the human equivalent dose of $50 \mathrm{mg} / 60 \mathrm{~kg}(0.83$ $\mathrm{mg} / \mathrm{kg})=0.83 \times 12.3=10.2 \mathrm{mg} / \mathrm{kg}$ mouse dose, $\mathrm{a}$ conversion coefficient of 12.3 is used to account for differences in body surface area between a mouse and a human.

\section{Weight-loading whole-body vibration (WWBV) Protocol}

Mice in the WWBV+DHEA group underwent WWBV following the training protocol as shown in Figure 1. The vibration platform provided a frequency of $5.6 \mathrm{~Hz}$ (peak acceleration, $0.13 \mathrm{~g}$ ) and the peak-to-peak amplitude of the vibration was $2 \mathrm{~mm}$. Regardless of frequency, a vibration platform produces a gravitational force $<1 \mathrm{~g}$. The WWBV training was under continuous supervision for 15 $\mathrm{min} /$ day, 5 days/week for 4 weeks. Training sessions 
were regular, beginning at 9 am each day. WWBV weight loading at first week began at $5 \%$ body weight and gradually increased to $10 \%$ by the end of the first week. The weight loading increased step by step, increasing to $15 \%-20 \%$ body weight in the second week and $25 \%$ to $30 \%$ body weight in the third week. A load of $40 \%$ body weight was defined as the goal of the final loading weight during the fourth week in the training protocol. According to life phase equivalencies [18], all the mice that underwent the training protocol were between 36.5 to 38 years old by human age equivalence.

\section{Forelimb Grip Strength}

A low-force testing system (Model-RX-5, Aikoh Engineering, Nagoya, Japan) was used to measure the forelimb grip strength of the mice. The amount of tensile force was measured by use of a force transducer equipped with a metal bar ( $2 \mathrm{~mm}$ diameter and $7.5 \mathrm{~cm}$ long). The detailed procedure has been described in our previous study [19]. Forelimb grip strength was tested after consecutive administration of SC, DHEA and DHEA + WWBV treatments for 4 weeks and $1 \mathrm{~h}$ after the last treatment. The maximal force (in grams) recorded by this low-force system was used as the grip strength.

\section{Swimming Exercise Performance Test}

Mice were subjected to the different treatments (SC, DHEA and WWBV+DHEA) for 4 weeks, followed by an exhaustive swimming test after the last treatment. The details of the exhaustive swimming test were described previously [19]. The endurance of each mouse was recorded as the time from the beginning to the time of exhaustion. Exhaustion was determined by observing the loss of coordinated movements and failure to return to the surface within $7 \mathrm{~s}$.

\section{Determination of Blood Biochemical Variables}

The effect of 4-weeks treatment (SC, DHEA and WWBV+DHEA) on serum lactate, ammonia, glucose, blood urea nitrogen (BUN) and creatine kinase (CK) levels in the mice was evaluated post-exercise. At $1 \mathrm{~h}$ after the final administration, a 15-min swimming test was performed without weight loading. Blood samples were immediately collected from the submandibular duct of the mice and centrifuged at $1500 \times g$ and $4{ }^{\circ} \mathrm{C}$ for $10 \mathrm{~min}$ for serum preparation. Levels of lactate, ammonia, glucose, CK and BUN in the serum were determined by using an autoanalyzer (Hitachi 7060, Hitachi, Tokyo). At the end of the experiment, all mice were euthanasia by $95 \% \mathrm{CO}_{2}$ asphyxiation and blood was withdrawn by cardiac puncture and all tissues (liver, skeletal muscle, heart, kidney, lung, epididymal fat pad tissue and brown adipose tissue) were collected and weighted. Serum was collected by centrifugation and levels of testosterone, aspartate aminotransferase (AST), alanine aminotransferase (ALT), creatinine (CREA), $\mathrm{CK}$, blood urea nitrogen (BUN), albumin, glucose and total triglyceride (TG) were assessed by an autoanalyzer (Hitachi 7060).

\section{Tissue Glycogen Determination}

Liver and muscle tissues were investigated to determine if SC, DHEA or WWBV+DHEA treatment increased glycogen deposition. About $1 \mathrm{~h}$ after the last administration of treatment, mice were euthanized by $\mathrm{CO}_{2}$ inhalation. The liver was excised and weighed. The method of glycogen analysis has been described in our previous studies $[19,20]$.

\section{Statistical analysis}

All data were expressed as mean \pm SEM $(n=8)$. Differences between groups were analyzed by one-way ANOVA using Duncan's post-hoc test and p values $<0.05$ were considered significant.
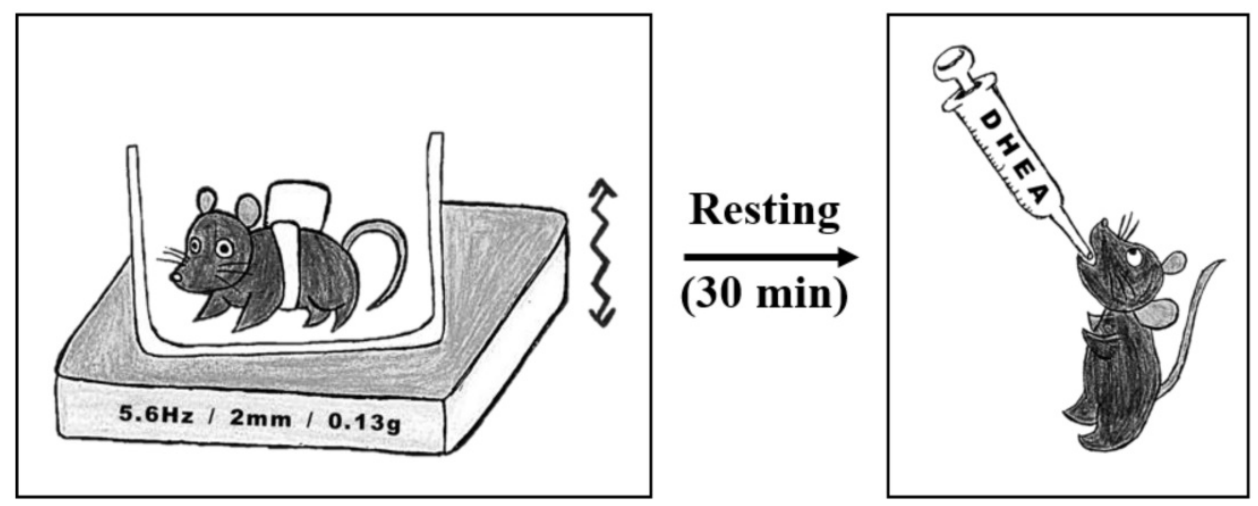

Figure 1. Protocol for 4-weeks weight-loading whole-body vibration training (WWBV). The DHEA supplementation was given 30 minutes after completion of the WWBV training. The loading weight was gently increase each week from $5 \%$ in the beginning to $40 \%$ in the final week. 


\section{Results}

\section{Effect of DHEA Supplementation Combined with WWBV Training on Body Weight (BW) and Other Metabolism-related Organ Weights}

The initial BWs of the SC, DHEA and WWBV+DHEA groups were $32.4 \pm 0.5,32.6 \pm 0.6$ and $32.3 \pm 0.3$ grams respectively, with no difference among the groups (Figure 2). At the end of the experiment, the $\mathrm{BW}$ in the SC, DHEA and WWBV+DHEA groups were $31.2 \pm 0.6,31.3 \pm 0.6$ and $31.3 \pm 0.3$ grams. All the middle-aged mice showed no significant difference in BW with the DHEA supplementation or WWBV training program. During the experimental period, body weight decreased slightly in all groups. This result is similar to our previous study, whereby the middle-aged mice showed slightly decreased BW during the experimental period (Lin et al., 2015). The effects of 4-week treatment (SC, DHEA and WWBV+DHEA) on body weight, food intake, water intake and tissue changes are shown in Table 1. The diet and water intakes of the DHEA and WWBV+DHEA groups were significantly higher by 1.04-fold $(p=0.0434)$ and 1.09-fold $(p=0.0048)$ respectively, than the SC group. In addition, there was no significant change in the kidney, lung, muscle, heart, and weight of brown adipose tissue (BAT). The epididymal fat pad (EFP) weight of the WWBV+DHEA group was significantly lower by $33.96 \%(p=0.0027)$ than the SC group. We also calculated the relative tissue weight $(\%)$, which is measured by the different tissue weights adjusted for individual $\mathrm{BW}$. The relative EFP weight of the WWBV+DHEA group decreased by $33.45 \%(p=$ 0.0040) compared to the SC group. Kidney, heart, lung, muscle and BAT relative weights did not differ among the three groups. Consistent with the results in Table 1, WWBV training + DHEA supplementation increased middle-aged mice appetite and decreased the white adipose tissue. Although the absolute and relative weight of the lung changed slightly in the WWBV+DHEA group, the values were still within reasonable limits. Taken together, our results show that the WWBV-training + DHEA supplementation regimen could be incorporated into the daily routine of mice without adverse effects on daily food and water consumption or on body weight. Instead, there are beneficial effects on body composition decreased fat accumulation.

\section{Effect of DHEA Supplementation Combined with WWBV Training on Forelimb Grip Strength and Endurance Swimming Performance}

The forelimb grip demonstrates the maximal muscle strength force production. The grip strength was $134 \pm 5,154 \pm 4$ and $155 \pm 2 \mathrm{~g}$ for the SC, DHEA and WWBV+DHEA groups, respectively (Figure 3 ). Compared with the SC group, the grip strength was significantly higher by 1.15 -fold $(p=0.0006)$ and 1.16-fold $(p=0.0003)$ in the DHEA group and WWBV+DHEA group, respectively. Exercise endurance is an important variable in evaluating aerobic capacity, with swim time to exhaustion reflecting the endurance exercise capacity. The exercise endurance levels in the swimming test for the SC, DHEA and WWBV+DHEA groups were 2.8 \pm 0.2 , $4.2 \pm 0.5$ and $5.2 \pm 0.6 \mathrm{~min}$, respectively (Figure 4 ). The swim time to exhaustion in the DHEA and WWBV+DHEA groups were significantly higher (1.47-fold, $p=0.0458)$ and (1.83-fold, $p=0.0013)$ than the SC group.

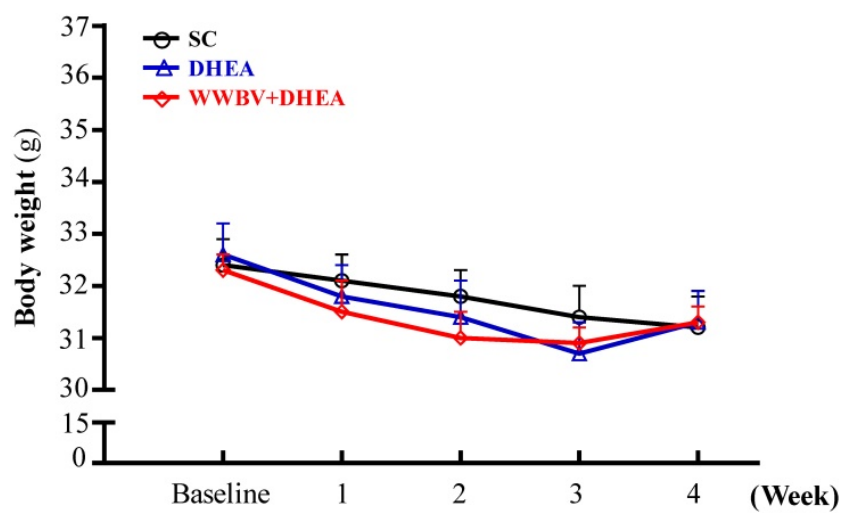

Figure 2. Effect of DHEA supplementation combined with WBV training on bodyweight (BW) for 4 weeks. Data was expressed as mean \pm SEM $(n=8)$.

Table 1. Effect on body weight, food intake, water intake and tissue changes on the 3 groups of mice (SC, DHEA and WWBV+DHEA) after 4 weeks.

\begin{tabular}{|c|c|c|c|}
\hline Characteristic & SC & DHEA & WWBV+DHEA \\
\hline Initial BW (g) & $32.4 \pm 0.5$ & $32.6 \pm 0.6$ & $32.3 \pm 0.3$ \\
\hline Final BW (g) & $31.2 \pm 0.6$ & $31.3 \pm 0.6$ & $31.3 \pm 0.3$ \\
\hline Food intake (g/day) & $3.66 \pm 0.08 \mathrm{a}$ & $3.77 \pm 0.04^{\mathrm{a}}$ & $3.82 \pm 0.06^{b}$ \\
\hline Water intake (mL/day) & $5.52 \pm 0.13^{a}$ & $5.68 \pm 0.12^{a}$ & $6.03 \pm 0.17 \mathrm{~b}$ \\
\hline Liver $(g)$ & $1.31 \pm 0.03 \mathrm{a}$ & $1.37 \pm 0.02^{\mathrm{a}}$ & $1.44 \pm 0.02^{\mathrm{b}}$ \\
\hline Kidney (g) & $0.36 \pm 0.02$ & $0.37 \pm 0.01$ & $0.36 \pm 0.02$ \\
\hline $\mathrm{EFP}(\mathrm{g})$ & $0.53 \pm 0.04^{a}$ & $0.53 \pm 0.05^{\mathrm{a}}$ & $0.35 \pm 0.02^{b}$ \\
\hline Heart (g) & $0.19 \pm 0.01$ & $0.19 \pm 0.01$ & $0.20 \pm 0.02$ \\
\hline Lung (g) & $0.18 \pm 0.01$ & $0.18 \pm 0.00$ & $0.18 \pm 0.01$ \\
\hline Muscle (g) & $0.35 \pm 0.01$ & $0.35 \pm 0.01$ & $0.35 \pm 0.01$ \\
\hline BAT (g) & $0.09 \pm 0.01$ & $0.10 \pm 0.01$ & $0.09 \pm 0.01$ \\
\hline Relative liver weight (\%) & $4.20 \pm 0.10^{a}$ & $4.39 \pm 0.11^{\mathrm{ab}}$ & $4.61 \pm 0.11^{b}$ \\
\hline $\begin{array}{l}\text { Relative Kidney weight } \\
(\%)\end{array}$ & $8.67 \pm 0.36$ & $8.48 \pm 0.61$ & $7.91 \pm 0.69 c$ \\
\hline Relative EFP weight (\%) & $1.71 \pm 0.16^{\mathrm{b}}$ & $1.69 \pm 0.15^{b}$ & $1.12 \pm 0.05^{a}$ \\
\hline Relative Heart weight (\%) & $0.60 \pm 0.04$ & $0.60 \pm 0.01$ & $0.63 \pm 0.07$ \\
\hline Relative Lung weight (\%) & $0.58 \pm 0.02$ & $0.58 \pm 0.02$ & $0.58 \pm 0.02$ \\
\hline $\begin{array}{l}\text { Relative Muscle weight } \\
(\%)\end{array}$ & $1.13 \pm 0.02^{b}$ & $1.11 \pm 0.02$ & $1.11 \pm 0.01$ \\
\hline Relative BAT weight (\%) & $0.29 \pm 0.02$ & $0.31 \pm 0.04$ & $0.30 \pm 0.02$ \\
\hline \multicolumn{4}{|c|}{$\begin{array}{l}\text { Data is expressed as mean } \pm \text { SEM }(n=8) \text {. Different letters indicate significant } \\
\text { difference at } p<0.05 \text { by one-way ANOVA. Muscle mass includes both } \\
\text { gastrocnemius and soleus muscles at the back part of the lower legs. BW, body } \\
\text { weight; EFP, epididymal fat pad; BAT, brown adipose tissue; sedentary control } \\
\text { with vehicle (SC); DHEA supplementation (DHEA); weight-loading whole-body } \\
\text { vibration (WWBV) combined with DHEA supplementation (WWBV+DHEA). }\end{array}$} \\
\hline
\end{tabular}




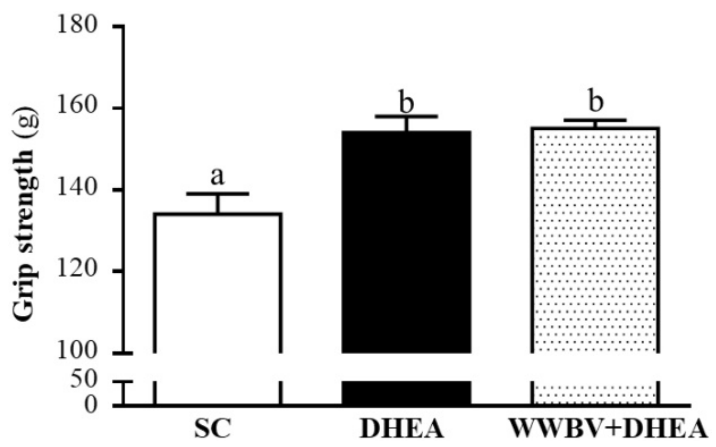

Figure 3. Effect of 4-weeks treatment (SC, DHEA and WWBV+DHEA) on forelimb grip strength (a) and swimming exercise performance (b). Male middle-age C57BL/6 mice underwent a grip strength test $1 \mathrm{~h}$ after the final administration of DHEA or WWBV training. For the swimming performance test, mice were first either pretreated with DHEA or the WWBV training. One hour later, the mice were subjected to an exhaustive swimming exercise with a load equivalent to $5 \%$ of the mouse's body weight attached to the tail. Data is expressed as mean \pm SEM $(n=8)$. Different letters indicate significant difference at $p<0.05$ by one-way ANOVA.

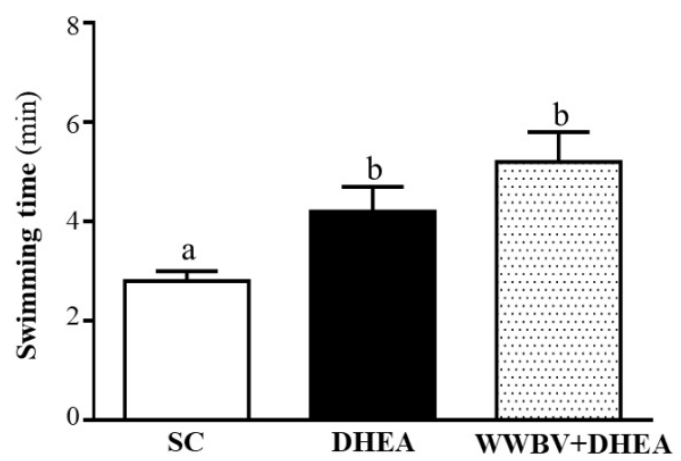

Figure 4. Effect of 4-weeks treatment (SC, DHEA, and WWBV+DHEA) on serum testosterone level. Data is expressed as mean $\pm \operatorname{SEM}(n=8)$. Different letters indicate significant difference at $p<0.05$ by one-way ANOVA.

\section{Effect of DHEA Supplementation Combined with WBV Training on Serum Lactate, Ammonia, Glucose, CK, and BUN Levels after Acute Exercise Challenge}

In the present study, lactate levels in the SC, DHEA and WWBV+DHEA groups were 6.1 $\pm 0.4,5.2 \pm 0.2$ and $4.1 \pm 0.2 \mathrm{mmol} / \mathrm{L}$. The lactate level was lowered by $14.72 \%(p=0.0261)$ for the DHEA treatment group and $32.31 \%(p<0.0001)$ for the WWBV+DHEA treatment group when compared with the SC group (Figure 5a). Although 4-weeks DHEA supplementation could decrease the serum lactate accumulation after acute exercise, WWBV training with DHEA supplementation was able to decrease the serum lactate accumulation better than with DHEA supplementation alone. Serum ammonia levels in the SC, DHEA and WWBV+DHEA groups were 141 \pm 31 , $93 \pm 13$ and $40 \pm 2 \mu \mathrm{mol} / \mathrm{L}$, respectively, with ammonia level of the WWBV+DHEA group lower by $71.66 \%(p$ $=0.0013$ ) compared with the SC group (Figure 5b). Blood glucose level is an important index for performance maintenance during exercise. Serum glucose levels in the SC, DHEA and WWBV+DHEA groups were $195 \pm 11,239 \pm 11$ and $265 \pm 6 \mathrm{mg} / \mathrm{dL}$, with serum glucose levels of DHEA and WWBV+DHEA groups significantly higher by 1.23 -fold $(p=0.0039)$ and 1.36-fold $(p<0.0001)$ than the SC group (Figure 5c). In this study, neither DHEA nor WBV training alone or in combination, has any beneficial effect on glucose levels after acute exercise. Serum CK is an important clinical biomarker for muscle damage, including muscular dystrophy, severe muscle breakdown, myocardial infarction, autoimmune myositis and acute renal failure. Serum CK activities of the SC, DHEA and WWBV+DHEA groups were $285 \pm 64,277 \pm 34$ and $162 \pm 31 \mathrm{U} / \mathrm{L}$, respectively (Figure 5d). The CK activity of each group was not significantly different (Figure 4d). The serum BUN levels of the SC, DHEA and WWBV+DHEA groups were $29.8 \pm 0.6,27.6 \pm 0.9$ and $21.1 \pm 0.7 \mathrm{mg} / \mathrm{dL}$, respectively. The BUN levels of the DHEA and WWBV+DHEA groups were significantly lower by $7.54 \%(p=0.0370)$ and $29.20 \%(p<0.0001)$ than the SC group (Figure 4e). Our results indicate that DHEA supplementation alone could reduce serum lactate and BUN levels, as well as increase glucose levels after acute exercise challenge. The addition of WWB to DHEA ameliorates the lactate, ammonia and BUN accumulation and optimizes glucose utilization after acute exercise better than with DHEA supplementation alone. Our results show that the WWBV training program in combination with DHEA, an ergogenic supplement, is able to aid fatigue recovery after acute exercise challenge in middle-aged mice.

Table 2. Effect on biochemical serum levels on the 3 groups of mice (SC, DHEA and WWBV+DHEA) after 4 weeks.

\begin{tabular}{llll}
\hline Parameter & SC & DHEA & DHEA + WBV \\
\hline $\begin{array}{l}\text { Testoterone } \\
\text { (ng/mL) }\end{array}$ & $12.8 \pm 1.5^{\mathrm{a}}$ & $16.0 \pm 0.0^{\mathrm{b}}$ & $16.0 \pm 0.0^{\mathrm{b}}$ \\
AST (U/L) & $115.0 \pm 11.3$ & $114.4 \pm 13.0$ & $83.9 \pm 9.7$ \\
ALT (U/L) & $73.8 \pm 8.7 \mathrm{a}$ & $72.8 \pm 8.3^{\mathrm{a}}$ & $51.9 \pm 4.5^{\mathrm{b}}$ \\
Creatinine & $0.30 \pm 0.01$ & $0.29 \pm 0.02$ & $0.29 \pm 0.01$ \\
(mg/dL) & & & \\
CK $(\mathrm{U} / \mathrm{L})$ & $518 \pm 89$ & $333 \pm 56$ & $309 \pm 90$ \\
BUN $(\mathrm{mg} / \mathrm{dL})$ & $21.7 \pm 1.0 \mathrm{a}$ & $16.1 \pm 0.6 \mathrm{~b}$ & $16.1 \pm 0.8 \mathrm{~b}$ \\
Albumin (g/dL) & $3.29 \pm 0.02$ & $3.30 \pm 0.04$ & $3.35 \pm 0.10$ \\
Glucose (mg/dL) & $192.0 \pm 9.6$ & $190.9 \pm 8.5$ & $195.3 \pm 4.9$ \\
TG(mg/dL) & $89.3 \pm 6.7 \mathrm{a}$ & $81.5 \pm 1.9 \mathrm{~b}$ & $75.1 \pm 4.9 \mathrm{~b}$
\end{tabular}

Data is expressed as mean \pm SEM $(n=8)$. Different letters indicate significant difference at $p<0.05$ by one-way ANOVA. AST, aspartate aminotransferase; ALT, alanine aminotransferase; BUN, blood urea nitrogen; $\mathrm{CK}$, creatine kinase; total triglyceride, TG.

\section{Effect of DHEA Supplementation Combined with WWBV Training on the Serum Testosterone Level}

The serum testosterone levels of the SC, DHEA and WWBV+DHEA groups were 12.8 $44.3,16.0 \pm 0.0$ 
and $16.0 \pm 0.0 \mathrm{ng} / \mathrm{mL}$, respectively (Table 2 ). In the DHEA and WWBV+ DHEA group, the testosterone levels were both significantly increased by 1.25 -fold ( $p$ $=0.0173$ and $p=0.0174$ ) compared with the SC group. There was no significant difference between the DHEA and WWBV+DHEA groups. This result demonstrates that WWBV in combination with DHEA supplementation, could increase the testosterone level.

\section{Effect of DHEA Supplementation Combined with WWBV Training on Biochemical Analyses}

Further biochemical analyses were carried out at the end of the experiments. The levels of AST,

(a)

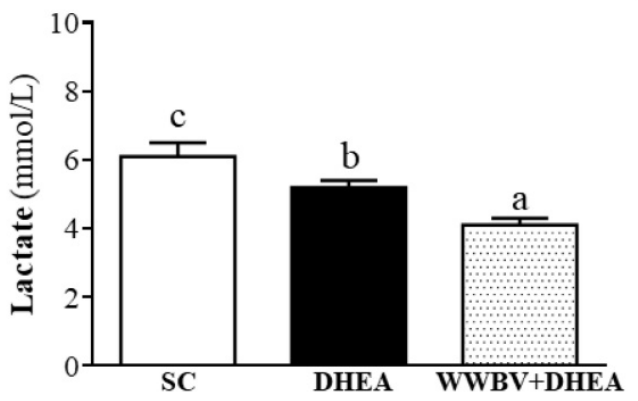

(c)

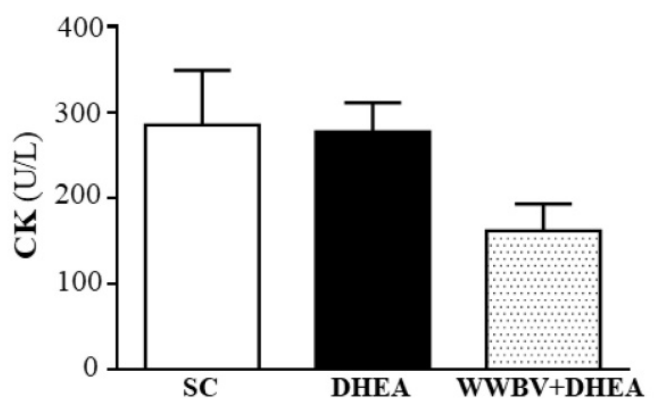

creatinine, CK, albumin and glucose did not differ significantly among the groups (Table 2), further substantiating the lack of adverse effects on the biochemical markers in the WWBV+DHEA group. In addition, level of ALT was significantly lowered (29.66\% decrease, $p=0.05)$ in the WWBV+DHEA group compared with the SC group. Similarly, the TG level of WWBV+DHEA group was significantly lower $(15.83 \%, p=0.0017)$ than the SC group. Taken together, this indicates that DHEA supplementation combined with WWBV training, may have a potential role in protecting the liver and decreasing lipid accumulation in middle-aged mice.

(b)

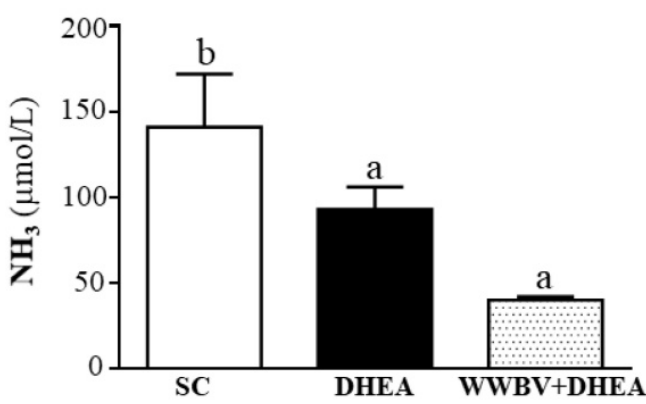

(d)

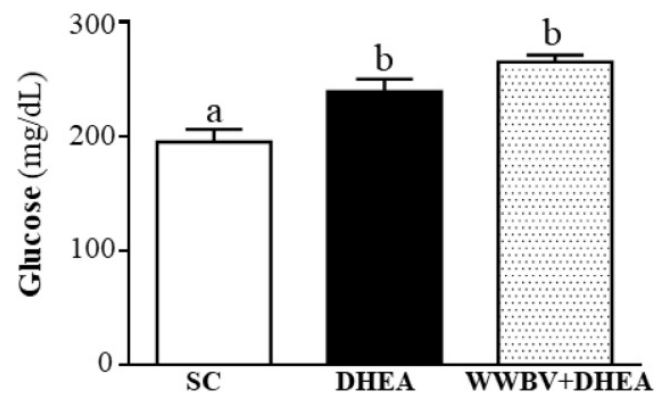

(e)

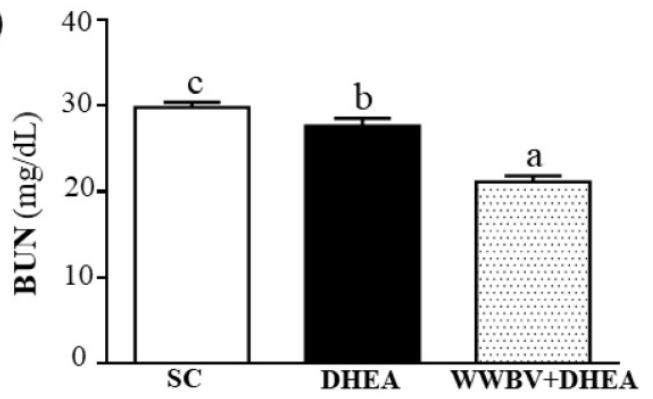

Figure 5. Effect of 4-weeks treatment (SC, DHEA and WWBV+DHEA) on serum levels of (a) lactate, (b) ammonia ( $\left.\mathrm{NH}_{3}\right)$, (c) glucose, (d) creatine kinase (CK), and (e) blood urea nitrogen (BUN) after an acute exercise challenge. One hour after the final administration of SC, DHEA or WWBV+DHEA, the mice performed a 15 -min swimming test without weight loading. Data is expressed as mean \pm SEM $(n=8)$. Different letters indicate significant difference at $p<0.05$ by one-way ANOVA. 
(a)

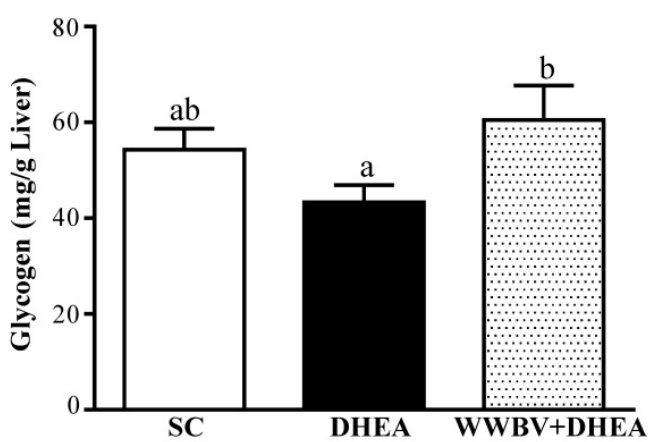

(b)

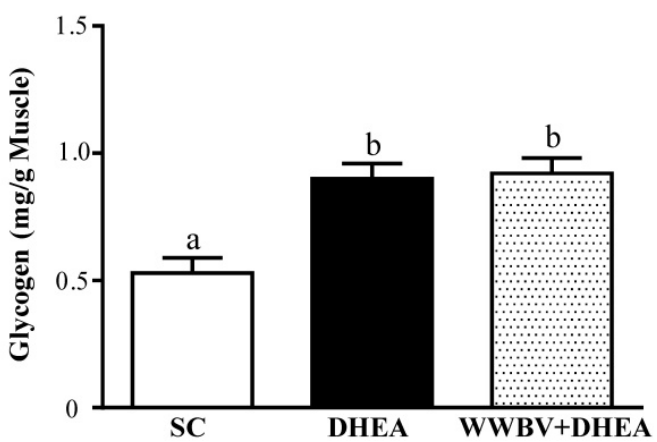

Figure 6. Effects after 4-weeks treatment (SC, DHEA and WWBV+DHEA) on (a) hepatic glycogen and (b) muscle glycogen levels at rest. Data is expressed as mean $\pm \operatorname{SEM}(n=8)$. Different letters indicate significant difference at $p<0.05$ by one-way ANOVA

\section{Effect of DHEA Supplementation Combined with WWBV Training on Hepatic and Muscle Glycogen Level}

Glycogen is the predominant source of glycolysis [20]. During high-intensity exercise, muscle obtains enough energy from anaerobic glycolysis and lactate is produced through glycolysis [19]. The glycogen content of the liver tissues is shown in Figure 6a. The liver glycogen levels of the SC, DHEA and WWBV+DHEA groups were 54.3 $\pm 4.4,43.3 \pm 3.6$ and $60.5 \pm 7.2 \mathrm{mg} / \mathrm{g}$, respectively. The liver glycogen content of the WWBV+DHEA group was 1.4-fold higher $(p=0.0307)$ than the group with DHEA supplementation alone. SC group and DHEA group showed no significant difference in liver glycogen levels. The glycogen content of muscle tissues in the SC, DHEA and WWBV+DHEA groups were $0.53 \pm 0.06,0.90 \pm 0.06$ and $0.92 \mathrm{e} \pm 0.06 \mathrm{mg} / \mathrm{g}$ muscle, respectively. Compared with SC group, muscle glycogen levels of DHEA group and WWBV + HEA group were significantly increased by 1.71 -fold $(p=$ $0.0002)$ and 1.76-fold $(p=0.0001)$ (Figure 6b). Taken together, these results indicate that 4-weeks WWBV training in male middle-aged mice could increase glycogen storage both in liver and muscle, giving rise to better glycogen storage levels than with DHEA supplementation alone.

\section{Histopathological Evaluation and Immuno- histochemistry (IHC) of gastrocnemius muscles}

Figure 7 shows the histological observations of the liver, muscle, heart, kidney, lung, testes and BAT in all 3 groups (SC, DHEA and WWBV+DHEA) taken at the end of the experiment. Results indicate that there were no histopathological differences among the groups. Additionally, the histopathological examinations showed that DHEA or WWBV+DHEA treatment did not result in any toxic effects on the major organs such as the liver, skeletal muscles, heart or kidney.

\section{Comment}

Frailty is considered highly prevalent in old age and confers a high risk for falls. Regular resistance training (two or three nonconsecutive days/week) can increase muscle mass in adults of all ages through to the 10th decade of life and is the best way to prevent frailty [21]. Based on these reason, we developed the weight-loading whole-body vibration (WWBV) training method to achieve the same effect as resistance training. Muscle tissue is the primary site for glucose and triglyceride disposal, so muscle loss specifically increases the risk of glucose intolerance and associated health issues [3,22]. In this study, we combined WWBV training with DHEA treatment since WWBV training is easier to learn than traditional resistance training.

Stimulated resistance training decreases adipose tissue. Some research has revealed significant reductions in intra-abdominal fat resulting from resistance training in older women [23] and older men [24, 25]. Herein, we investigated whether WWBV training also has the same effect as resistance training in lowering body fat mass. WWBV training has the advantage of being easier to learn than traditional strength training among the aging population. We found that WWBV+DHEA treatment showed obvious improvement in muscle strength, better than combining aerobic exercise with DHEA supplementation, which only improved lower extremity muscle strength in women in a previous study [26]. In our previous work, we found that DHEA supplementation in combination with normal WBV, was not able to improve exercise performance in young mice model [27]. Based on these findings, we conclude that DHEA is ineffective in young male mice model and only provides a slight effect on postmenopausal women. However, DHEA has an obvious effect on the middle-aged or older mouse model to increase exercise performance, especially in combination with an exercise training program [28, 29]. 
(a)

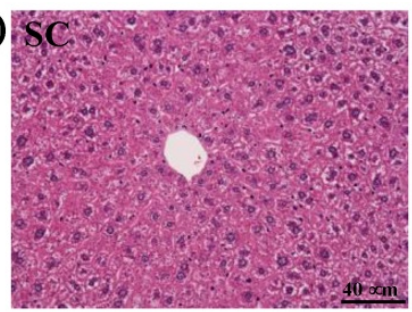

(b)

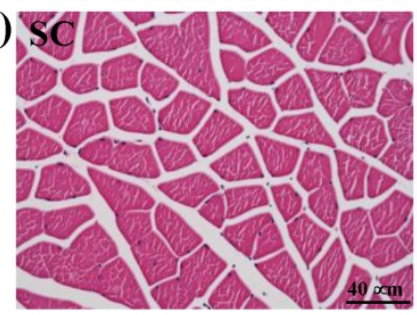

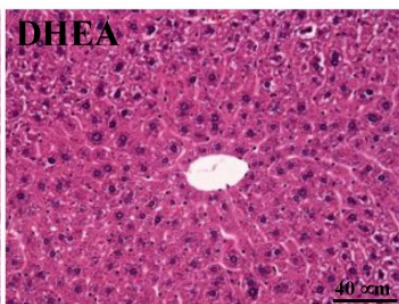

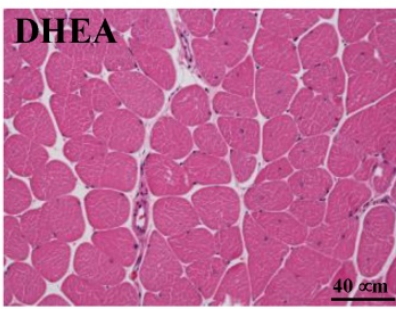

(c)

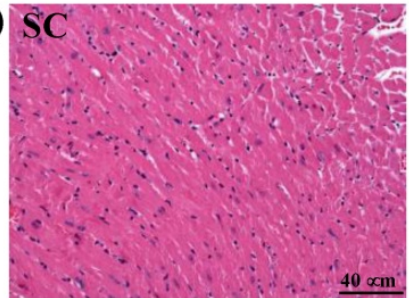

(d)

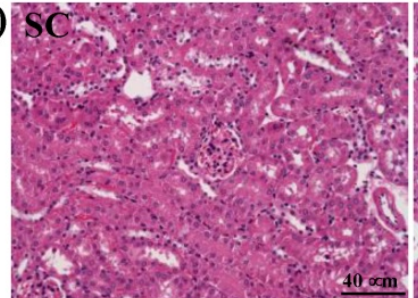

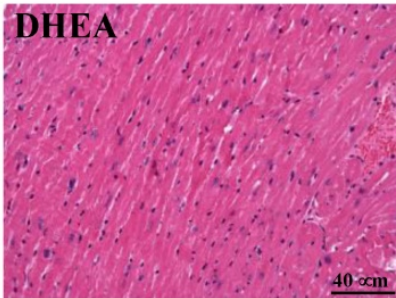

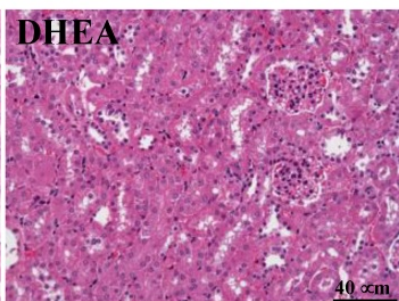

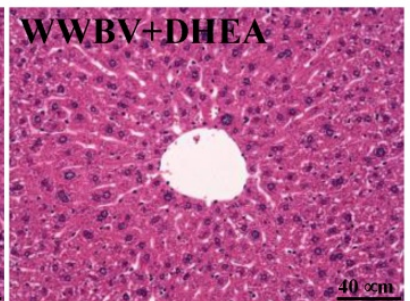
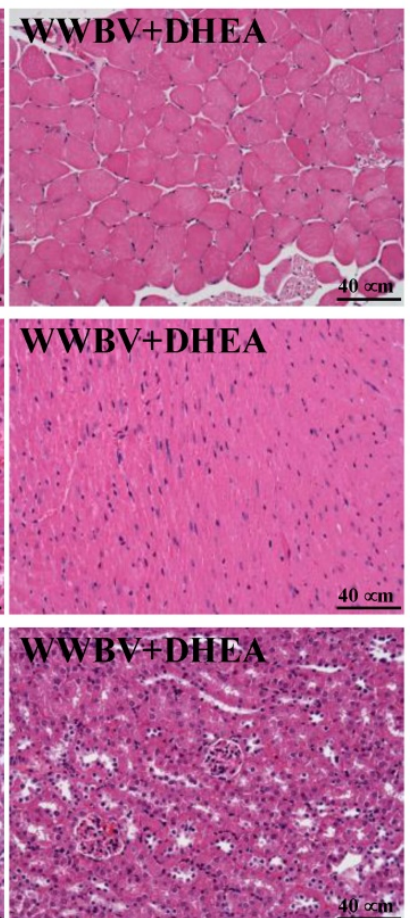

(e)

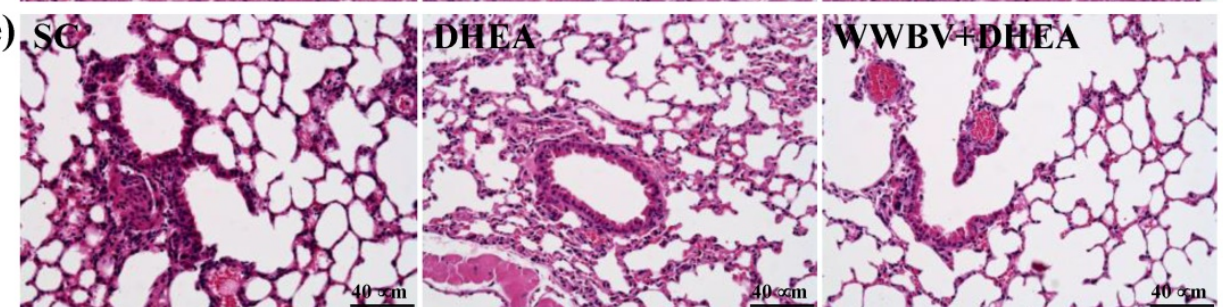

(f) $\mathrm{SC}$

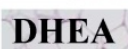

(g)

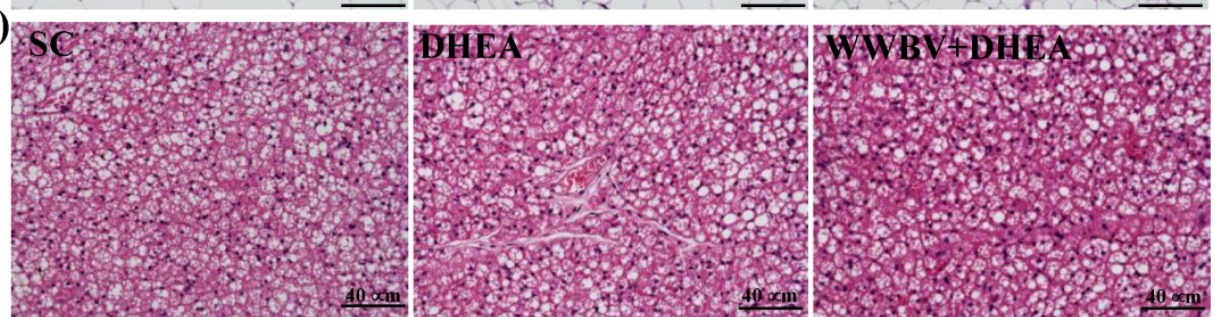

Figure 7. The H\&E staining of 4-weeks treated mice (SC, DHEA and WWBV+DHEA) on the morphology of (a) liver, (b) skeletal muscle, (c) heart, (d) kidney, (e) lung, (f) epididymal fat pad (EFP) tissue and (g) brown adipose tissue (BAT). Specimens were photographed with a light microscope (Olympus BX51). (Magnification: $\times 200$, Scale bar, $40 \mu \mathrm{m}$ ) 
We were able to demonstrate that WWBV+ DHEA treatment could improve fatigue indexes after acute exercise. Muscle fatigue after exercise was evaluated by multiple biochemical indicators, including lactate, ammonia, glucose, CK, and BUN levels [30]. Lactate is an oxidizable substrate in skeletal muscle and a precursor to gluconeogenesis in muscles or liver after exercise [31]. Ammonia, an important metabolite during energy metabolism for exercise, is generated by different sources. During exercise, increase of ammonia accumulation in the blood and brain can negatively affect the central nervous system and cause fatigue [32]. The maintenance of steady levels of blood glucose during physical exercise could extend exercise duration and improve exercise performance [33]. Urea is formed by the liver and carried by the blood to the kidneys and urea level is an important index correlating with protein breakdown, dehydration, stress and fatigue [34]. The present data suggests that WWBV+DHEA treatment for four weeks could decrease lactate, ammonia and BUN accumulation, as well as economize serum glucose utilization. Taken togther, the findings indicate that WWBV+DHEA can ameliorate exercise fatigue and promote recovery.

Several studies have shown improved lipoprotein-lipid profiles resulting from resistance training [35, 36], whereas other studies did not demonstrate significant changes in blood lipid levels [21]. In our previous study, we found that WBV could prevent high fat diet (HFD)-induced obesity and improve blood lipid profiles [37]. However, other investigators using resistance training showed a lack of effect on the blood lipid profiles [38]. We had also previously demonstrated that WBV without weight-loading or DHEA supplementation could not significantly change the blood lipid profiles in non-obese middle-aged mice [39]. DHEA is available in a supplement form and is a steroid hormone that is sometimes used to increase testosterone levels. Testosterone has been shown to induce glycogen synthesis [40]. From our results, we find that WWBV+DHEA treatment increases testosterone level in middle-aged mice. The consumption of carbohydrates varies according to the duration of the exercise. One of the factors restricting the synthesis of glycogen is the delivery of glucose through the cell membrane. After endurance and resistance exercise, muscle cells increase their glucose permeability and synthesis of glycogen, resulting in increased sensitivity of muscle to insulin [41]. According our previous results, DHEA supplementation could increase liver and muscle glycogen synthesis [27]. Our study suggests that after WWBV training, DHEA intake could increase liver and muscle glycogen synthesis within $30 \mathrm{~min}$. This may be of particular importance in the maintenance of glucose homeostasis via regulation of glycogen metabolism, hepatic glucose transport, as well as glucose uptake and utilization in the skeletal muscle [42, 43]. In our study, we see that the improvement on exercise performance in the WWBV+ DHEA group was clearly dependent on increasing glycogen storage.

In conclusion, we found that WWBV+DHEA treatment decreased white adipose tissue accumulation while increasing serum testosterone and glycogen (liver and muscle) levels. Furthermore, the WWBV+DHEA group had increase anti-fatigue activity after acute exercise. Plasma lactate, ammonia and BUN levels decreased, while serum glucose levels increased, all of which contribute to enhancing exercise performance in middle-aged mice. To our knowledge, this is the first study investigating WWBV+DHEA treatment in exercise performance, demonstrating increased glycogen (liver and muscle) storage content and increased anti-fatigue activity in middle-aged mice. This study suggests that WWBV could be a potential training method for use as a form of resistance training in middle-age adults.

\section{Acknowledgments}

This study was supported by the Ministry of Science and Technology of Taiwan (grant no. MOST-105-2410-H-255-001 to Wen-Chyuan Chen); Aileen Lim Ai Lin for her careful reading of the manuscript. The authors are grateful to Chien-Chao Chiu for the pathological examinations.

\section{Author Contributions}

Yi-Ming Chen, Wen-Chyuan Chen and Chi-Chang Huang designed the experiments. Wen-Ching Tseng and Yi-Ming Chen carried out the laboratory experiments. Yi-Ming Chen, Hao-Chieh Lee, Wen-Chyuan Chen and Chi-Chang Huang analyzed the data, interpreted the results, prepared figures, and wrote the manuscript. Wen-Chyuan Chen, Yi-Ming Chen and Chi-Chang Huang revised the manuscript. Chen Mu-Tsung and Wen-Chyuan Chen contributed DHEA, reagents, materials and analysis platforms.

\section{Competing Interests}

The authors have declared that no competing interest exists.

\section{References}

1. Newton RU, Häkkinen K, Häkkinen A, McCormick M, Volek J, Kraemer WJ. Mixed-methods resistance training increases power and strength of young and older men. Med Sci Sports Exerc. 2002; 34(8): 1367-1375.

2. MithalA, Bonjour JP, Boonen S, Burckhardt P, Degens H, Fuleihan GEH, Yoshimura N. Impact of nutrition on muscle mass, strength, and performance in older adults. Osteoporos Int. 2013; 24(5): 1555-1566. 
3. Flack KD, Davy KP, Hulver MW, et al. Aging, resistance training, and diabetes prevention. J Aging Res. 2010; 2011: 127315.

4. Phillips SM, Winett RA. Uncomplicated resistance training and health related outcomes: evidence for a public health mandate. Curr Sports Med. Rep. 2010; 9: 208-13.

5. Malafarina V, Úriz-Otano F, Iniesta R, Gil-Guerrero L. Sarcopenia in the elderly: diagnosis, physiopathology and treatment. Maturitas 2012; 71(2): 109-114

6. Baulieu EE, Robel P. Dehydroepiandrosterone (DHEA) and dehydroepiandrosterone sulfate (DHEAS) as neuroactive neurosteroids. Proc Natl Acad Sci U S A. 1998; 95: 4089-4091.

7. Belanger A, Candas B, Dupont A, Cusan L, Diamond P, Gomez JL, Labrie F. Changes in serum concentrations of conjugated and unconjugated steroids in 40- to 80-year old men. J Clin Endocrino. Metab. 2005; 79: 1086-1090.

8. Sato K, Iemitsu M, Aizawa K, Ajisaka R. Testosterone and DHEA activate the glucose metabolism-related signaling pathway in skeletal muscle. Am J Physiol Endocrinol Met. 2008; 294:E961-E968.

9. Sato K, Iemitsu M, Aizawa K, Mesaki N, Ajisaka R, Fujita S. DHEA administration and exercise training improves insulin resistance in obese rats. Nutr Metab. 2012; 9: 47

10. Coza A, Nigg BM, Dunn JF. Effects of vibrations on gastrocnemius medialis tissue oxygenation. Med Sci Sports Exerc. 2011; 43: 509-515.

11. Petit PD, Pensini M, Tessaro J, Desnuelle C, Legros P, Colson SS. Optimal whole-body vibration settings for muscle strength and power enhancement in human knee extensors. J Electromyogr Kinesiol. 2010; 20:1186-1195.

12. Verschueren SM, Roelants M, Delecluse C, Swinnen S, Vanderschueren D, Boonen S. Effect of 6-month whole body vibration training on hip density, muscle strength, and postural control in postmenopausal women: a randomized controlled pilot study. J Bone Miner Res. 2004; 19: 352-359.

13. Bogaerts A, Delecluse C, Claessens AL, Coudyzer W, Boonen S, Verschueren $\mathrm{SM}$. Impact of whole-body vibration training versus fitness training on muscle strength and muscle mass in older men: a 1-year randomized controlled trial. J Gerontol Ser A Biol Sci Med Sci. 2007; 62: 630-635.

14. Lau RW, Liao LR, Yu F, Teo T, Chung RC, Pang MY. The effects of whole body vibration therapy on bone mineral density and leg muscle strength in older adults: a systematic review and meta-analysis. Clin Rehabil. 2011; 25: 975-988.

15. Vissers D, Verrijken A, Mertens I. Effect of long-term whole body vibration training on visceral adipose tissue: a preliminary report. Obes Facts. 2010; 3: 93-100.

16. Domingos LL, Giehl PM, Paiva DN, Asad NR, Marin PJ, Bernardo-Filho M. Alterations on the plasma concentration of hormonal and non hormonal biomarkers in human beings submitted to whole body vibration exercises. Sci Res Essays. 2015; 10: 287-297.

17. [Internet] Guidance for Industry on Estimating the Maximum Safe Starting Dose in Initial Clinical Trials for Therapeutics in Adult Healthy Volunteers. Department of Health and Human Services, Food and Drug Administration. https://www.federalregister.gov/articles/2005/07/22/ 05-14456/guidance-for-industry-on-estimating-the-maximum-safe-starting-d ose-in-initial-clinical-trials-for.

18. Flurkey K, Currer JM, Harrison DE. The Mouse in Aging Research. In: Fox JG, et al, editors. The Mouse in Biomedical Research; 2nd Edition. Burlington, MA: American College Laboratory Animal Medicine (Elsevier). 2007:637-672.

19. Chen YM, Lin CL, Wei L, Hsu YJ, Chen KN, Huang CC, Kao CH. Sake protein supplementation affects exercise performance and biochemical profiles in power-exercise-trained mice. Nutrients. 2016; 8: 106.

20. Greenberg CC, Jurczak MJ, Danos AM, Brady MJ. Glycogen branches out: New perspectives on the role of glycogen metabolism in the integration of metabolic pathways. Am J Physiol Endocrinol Metab. 2006; 291: E1-E8.

21. Westcott WL. Resistance training is medicine: effects of strength training on health. Curr Sports Med. Rep. 2012; 11(4): 209-216.

22. Strasser B, Schobersberger W. Evidence of resistance training as a treatment therapy in obesity. J Obes. 2011; 2011: 482564.

23. Hunter GR, Bryan DR, Wetzstein CJ. Resistance training and intra- abdominal adipose tissue in older men and women. Med Sci Sports Exerc. 2002; 34: $1025-8$.

24. Ibañez J, Izquierdo M, Argüelles I, Forga L, Larrión JL, García-Unciti M, Gorostiaga EM. Twice-weekly progressive resistance training decreases abdominal fat and improves insulin sensitivity in older men with type 2 diabetes. Diabetes Care 2005; 28(3): 662-667.

25. Treuth MS, Ryan AS, Pratley RE, Rubin MA, Miller JP, Nicklas BJ, Hurley BF. Effects of strength training on total and regional body composition in older men. J Appl Physiol. 1994; 77: 614-20.

26. Kenny AM, Boxer RS, Kleppinger A, Brindisi J, Feinn R, Burleson J.A. Dehydroepiandrosterone combined with exercise improves muscle strength and physical function in frail older women. J Am Geriatr Soc. 2010; 58(9): 1707-1714.

27. Chen WC, Chen YM, Huang CC, Tzeng YD. Dehydroepiandrosterone Supplementation Combined with Whole-Body Vibration Training Affects Testosterone Level and Body Composition in Mice. Int J Med Sci. 2016; 13(10): 730 .

28. Sattler FR, Castaneda-Sceppa C, Binder EF, Schroeder ET, Wang Y, Bhasin S, Colletti $\mathrm{P}$. Testosterone and growth hormone improve body composition and muscle performance in older men. J Clin Endocrinol Metab. 2009; 94(6): 1991-2001.
29. Baker WL, Karan S, Kenny AM. Effect of dehydroepiandrosterone on muscle strength and physical function in older adults: a systematic review. J Am Geriatr Soc. 2011; 59(6): 997-1002.

30. Chen YM, Tsai YH, Tsai TY, Chiu YS, Wei L, Chen WC, Huang CC. Fucoidan supplementation improves exercise performance and exhibits anti-fatigue action in mice. Nutrients. 2014; 7: 239-252.

31. Brooks GA. Intra-and extra-cellular lactate shuttles. Med Sci Sports Exerc. 2000; 32: 790-799.

32. Ma GD, Chiu CH, Hsu YJ, Hou CW, Chen YM, Huang CC. Changbai Mountain ginseng (Panax ginseng CA Mey) extract supplementation mproves exercise performance and energy utilization and decreases fatigue-associated parameters in mice. Molecules. 2017; 22(2): 237.

33. Febbraio MA, Chiu A, Angus DJ, Arkinstall MJ, Hawley J. A. Effects of carbohydrate ingestion before and during exercise on glucose kinetics and performance. J Appl Physiol. 2000; 89(6): 2220-2226.

34. Wang JJ, Shieh MJ, Kuo SL, Lee CL, Pan TM. Effect of red mold rice on antifatigue and exercise-related changes in lipid peroxidation in endurance exercise. Appl Microbiol Biotechnol. 2006; 70: 247-253.

35. Kelley G, Kelley K. Impact of progressive resistance training on lipids and lipoproteins in adults: a meta-analysis of randomized controlled trials. Prev Med. 2009; 48: 9-19.

36. Tambalis K, Panagiotakos D, Kavouras S, Sidossis L. Responses of blood lipids to aerobic, resistance and combined aerobic with resistance exercise training: a systematic review of current evidence. Angiology. 2009; 60: 614-632.

37. Huang CC, Tseng TL, Huang WC, Chung YH, Chuang HL, Wu JH. Whole-body vibration training effect on physical performance and obesity in mice. Int J Med. Sci. 2014; 11(12): 1218

38. Smutok M, Reece C, Kokkinos $\mathrm{P}$, et al. Aerobic vs. strength training for risk factor intervention in middle-aged men at high risk for coronary heart disease. Metabolism. 1993; 42: 177-84.

39. Lin CI, Huang WC, Chen WC, Kan NW, Wei L, Chiu Y S, Huang CC. Effect of whole-body vibration training on body composition, exercise performance and biochemical responses in middle-aged mice. Metabolism. 2015; 64(9): 1146-1156.

40. Shen $M$, Shi $H$. Sex hormones and their receptors regulate liver energy homeostasis. Int J Endocrinol. 2015; 2015: 294278

41. Ivy JL. Muscle glycogen synthesis before and after exercise. Sports Med. 1991; 11: 6 .

42. Corona G, Monami M, Rastrelli G, Aversa A, Sforza A, Lenzi A, Maggi M. Type 2 diabetes mellitus and testosterone: A meta-analysis study. Int J Androl. 2011; 34: 528-540

43. Sato K, Iemitsu M. Exercise and sex steroid hormones in skeletal muscle. J Steroid Biochem Mol Biol. 2015; 145: 200-205. 